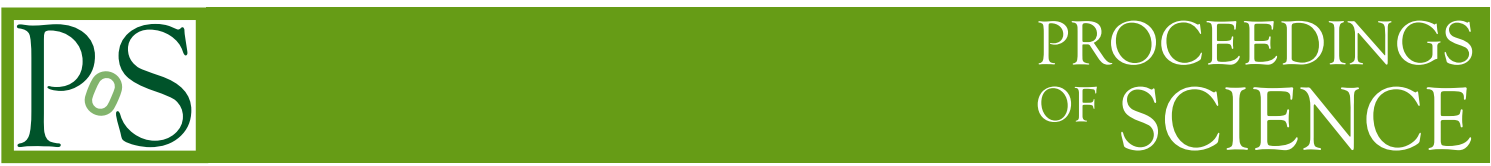

\title{
The LHCb muon detector at Upgrade II
}

\section{Francesco Debernardis $^{a, *}$}

${ }^{a}$ University and INFN, Bari (IT), on behalf of the LHCb Collaboration

$4 \quad$ E-mail: francesco.debernardis@cern.ch

The LHCb experiment is a $b$-physics dedicated experiment at the LHC collider. It has a wide physics program, covering different fields of interest: among others, precise measurements of the CKM matrix elements and the search for lepton flavour violation and beyond Standard Model physics. The $\mathrm{LHCb}$ detector has successfully performed during the Run 1 and Run 2 of the LHC, leading to important contributions in the field of flavour physics as well as physics in the forward region. Now, it is being upgraded in a first step - Upgrade I - to run at a luminosity of $2 \times 10^{33} \mathrm{~cm}^{-2} \mathrm{~s}^{-1}$. An Upgrade II phase has been proposed, aiming at a full exploitation of the flavour physics potential of the High Luminosity LHC operational period. LHCb Upgrade II will run at instantaneous luminosities of up to $2 \times 10^{34} \mathrm{~cm}^{-2} \mathrm{~s}^{-1}$ and accumulate a data sample corresponding to a minimum of $300 \mathrm{fb}^{-1}$. New design options for the muon detector at Upgrade II are under study, in order to deal with the increase in luminosity and readout rate, while preserving the stable operation of the detector and its highly efficient detection capability. Due to the high variability of the expected particle rates, ranging from several $\mathrm{kHz} / \mathrm{cm}^{2}$ in the external regions up to several $\mathrm{MHz} / \mathrm{cm}^{2}$ in the inner ones, different sub-detector technologies that could replace or simply complement the old ones are under investigation, with the aim to choose the best option in terms of granularity, radiation hardness and effective spark quenching up to integrated charges of $O\left(C / \mathrm{cm}^{2}\right)$. An overview of the state of the art of the muon detector design for the LHCb Upgrade II will be here presented.

The Ninth Annual Conference on Large Hadron Collider Physics online

7-12 June 2021

${ }^{*}$ Speaker 

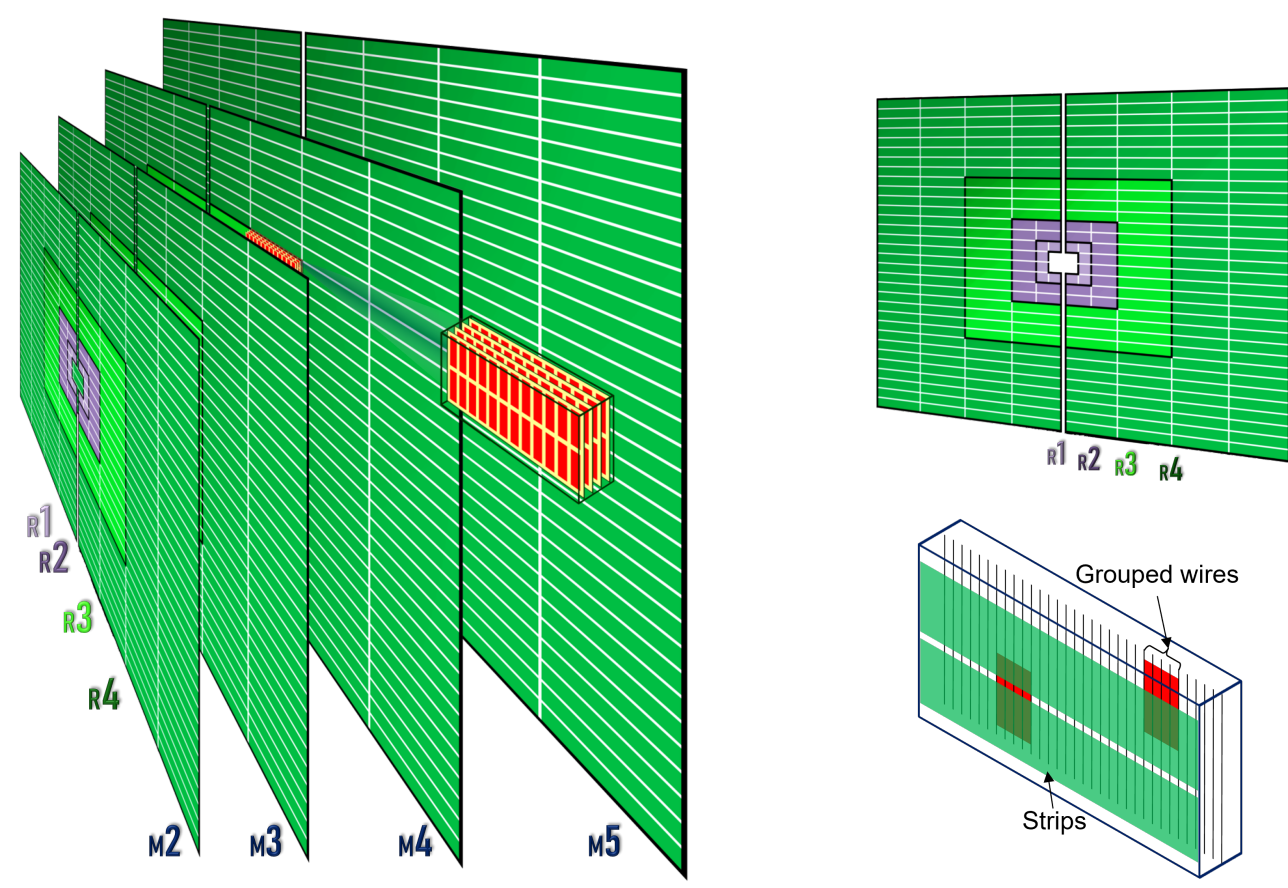

$R^{1} \mathrm{R} 2 \mathrm{R} 3 \mathrm{R} 4$

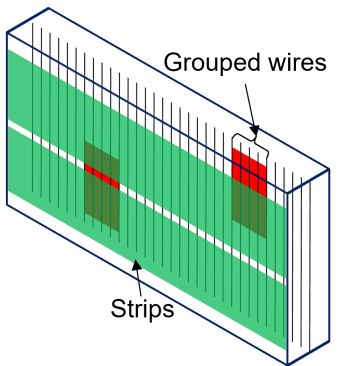

Figure 1: (Left) Current layout of the LHCb muon detector. An exploded view of a R3M4 chambers is shown, together with the detail of 4-gaps of (red) logical pads. (Top right) Frontal view of a station. (Bottom right) Sketch of logical pads. In each gap, logical pads are logical AND of grouped vertical wires with strips or physical pads.

\section{The LHCb muon detector and the Upgrade II}

The LHCb apparatus is a single-arm forward spectrometer covering a pseudorapidity range $82<\eta<5$, specifically designed for different fields of interest: among others, precise measurements of the CKM matrix elements and the search for lepton flavour violation and beyond Standard Model physics. The muon detector, located at the far end of the LHCb apparatus [1], consists of four rectangular stations ${ }^{1}$ (M2-M5) interleaved with $80 \mathrm{~cm}$ thick iron absorbers as shown in Fig. 1. The transverse dimensions of the stations scale with their distance from the $p p$ interaction point up to a $\sim 100 \mathrm{~m}^{2}$ maximum area of M5. All stations are divided into four regions (R1-R4) with increasing distance from the beam axis, all equipped with 4-gaps multi-wire proportional chambers (MWPCs). The LHCb Upgrade II is a great opportunity to improve the precision of the LHCb physics reach[3] by taking advantage of the LHC luminosity increase up to $2 \times 10^{34} \mathrm{~cm}^{-2} \mathrm{~s}^{-1}$ planned for Run 5 in 2032, two order of magnitude above the initial operating LHCb luminosity. In order to deal with the expected increase in the readout rate (a factor $\sim 10$ w.r.t Run 3 rates), the muon detector will be upgraded [4] preserving its highly efficient muon detection capability $>95 \%$ in a 25 ns time window. During LHC Run 5 the muon detector will be subjected to high particle rates together with an increased background and pileup expecially in the innermost R1-R2 regions where the

${ }^{1}$ A further station M1 was located upstream calorimeters during LHC Run 1 and Run 2 [2] but it was removed with the LHCb Upgrade I. 

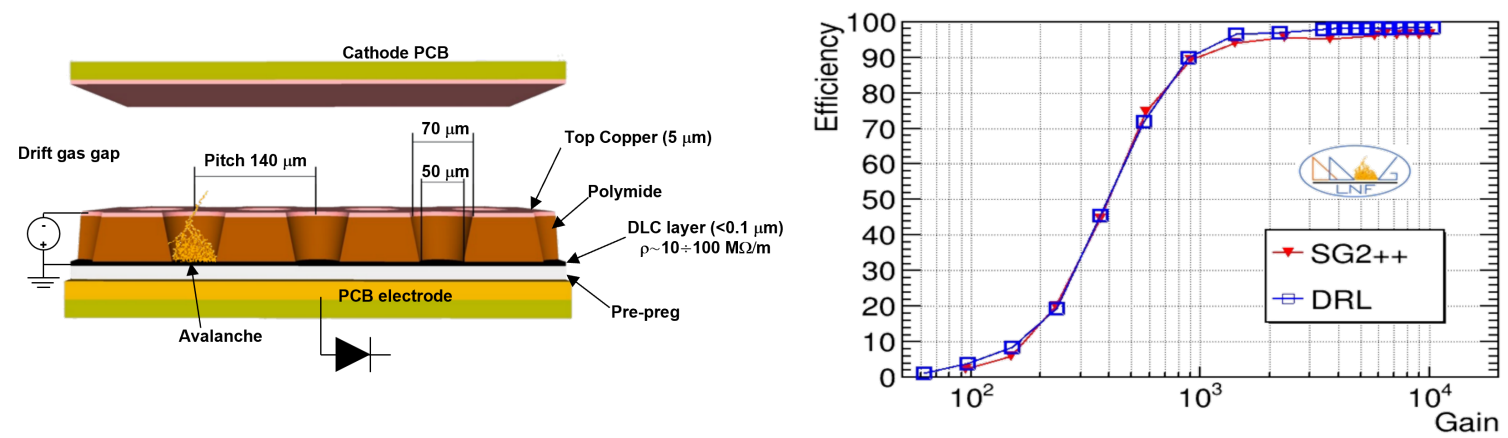

Figure 2: (Left) The exploded view of a $\mu$ RWELL detector [5]. (Right) The efficiency vs gain plot.

MWPCs need to be replaced. Instead, the MWPCs of the outer regions R3-R3 could be reused or replaced according to their state of aging. The replacement of the MWPCs is one of the crucial modification for the muon detector at Upgrade II. Some alternatives to MWPCs are under study and will be discussed in this proceeding. Another crucial improvement, in view of the muon detector performance optimisation, is a new redout scheme for the logical pads of the MWPCs that will be in use. This modification is thought for reducing background signals, as shown in the last section.

\section{Replacement options for the MWPCs}

During the Upgrade II phase, $\mu$ RWELLs are planned to replace the MWPCs in the inner regions R1-R2 of all stations, where particle rates will approach a maximum value of $1 \mathrm{MHz} / \mathrm{cm}^{2}$. Indeed this technology [5] has both higher rate capabilities and higher granularity with respect to the current MWPCs. $\mu$ RWELLs are novel micro pattern gaseous detector in which, the detection of charged particles is made with a matrix of blind holes (well), shown in Fig. 2. This matrix acts as an amplification stage generating avalanches from the gas ionization in the above drift gas gap. The holes of the matrix are blind with a highly resistive layer that suppresses the avalanche spark transition, allowing to achieve large gains. Thus, with the expected fluxes at Upgrade II the gains in the $\mu$ RWELLs can be $\geq 10^{4}$, guaranteeing an efficincy of $\sim 98 \%$ as shown in Fig. 2.

In the outer regions R3-R4 of the stations M2-M3 where particles rates will approach a maximum value of $13 \mathrm{kHz} / \mathrm{cm}^{2}$, the reuse plan for MWPCs depends on the aging tests results. In case of aging, RPCs and the SCI-Tiles are interesting alternatives to the MWPCs in R4, with maximum rates up to $\sim 10 \mathrm{kHz} / \mathrm{cm}^{2}$. The RPCs have high detector efficiency $\sim 99 \%$ and time resolution $\sim 1$ ns, as well as ease of construction, robustness and low cost per unit area. However this option is under study because, in order to preserve the detector performance at the expected rates at Upgrade II, the bulk resistivity of the electrodes and/or the electrode and gap thickness and/or the average charge per event must be reduced since the potential drop of the RPC gas gap depends on their product. Also the SCI-Tiles are an interesting option thanks to high detector efficiency $\sim 98 \%$ and a good time resolution $\sim 1 \mathrm{~ns}$, furthermore they are not expensive and easy to produce and assemble. The planned system consists of a Scintilling Tiles based detector, readout via Wave Lenght Shifting fibers (WLS) and Silicon Photo Multipliers (SiPM). The SiPM need to be replaced periodically and/or located in a neutron shielding and cooled down area on the back side of the tiles support, 


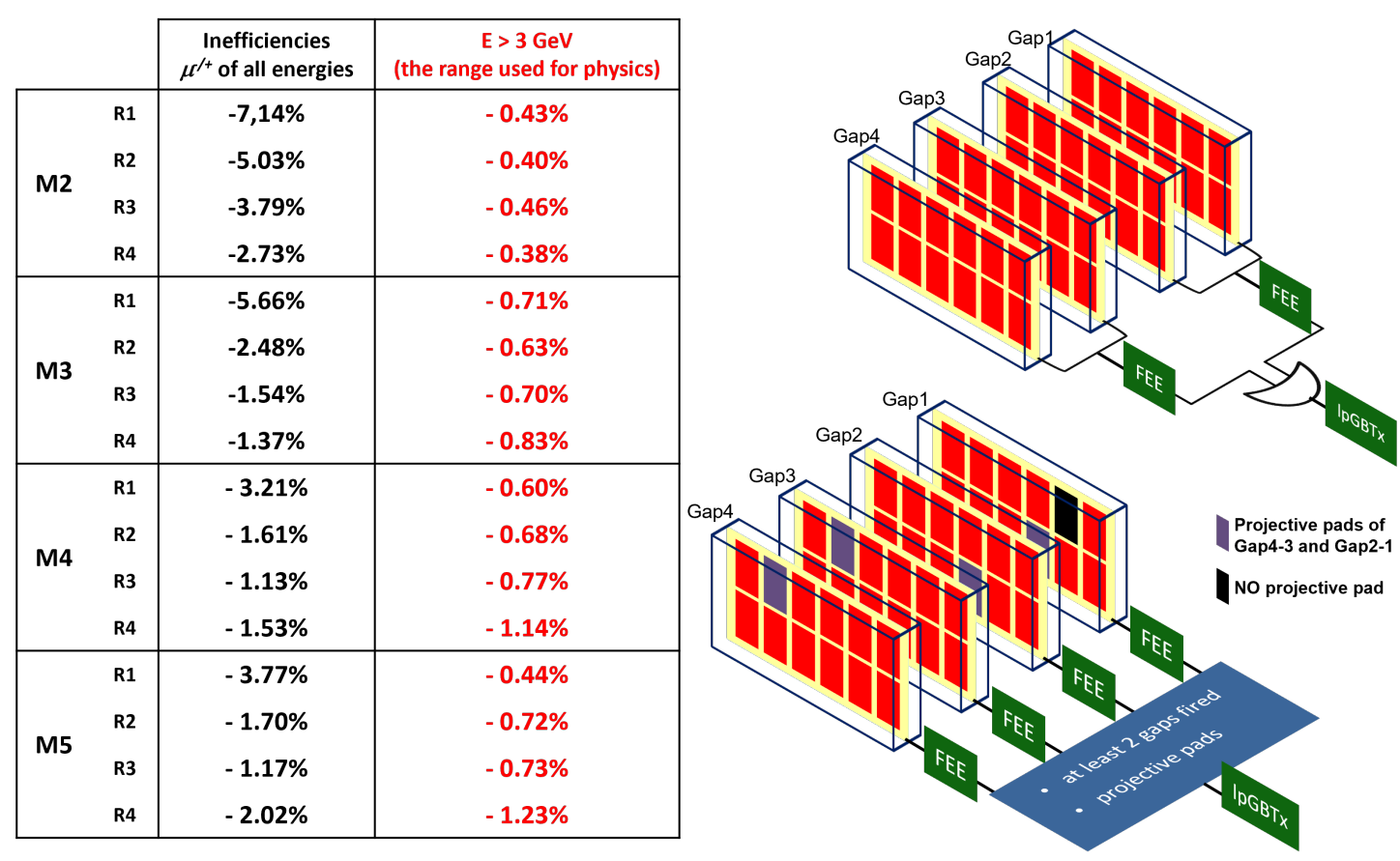

Figure 3: (Right) Current (top) and new (bottom) MWPC readout schemes are shown. (Left) Preliminary results on inefficiencies on muon identification due to the new readout scheme under study for MWPCs at Upgrade II.

since they deteriorate rapidly with the very harsh LHCb environment. In regions R3, the expected rates are too high for these technologies so that, in case of aging, it is planned to replace MWPCs with new ones.

\section{New readout scheme for MWPCs at Upgrade II}

The current readout scheme of one chamber consists in the OR of 2 gaps, again ORed at Front End Electronics (FEE) level, as shown in Fig. 3 (top right). This 4 gaps OR generates a very high input rate, up to $90 \%$ in inner regions, due to single gap background signal. In order to get rid of this effect, a new readout scheme has been proposed for Upgrade II (Fig. 3), in which each gap is readout separately requiring firstly signal in at least 2 gaps out of 4 of a chamber at the same time, and secondly at least two projective logical pads (Fig. 3) fired in the two gaps. Considering the effects of these modifications, the single gap readout is expected to reduce by $\sim 30 \div 40 \%$ the input rate to each FEE channel but the contribution from uncorrelated hits is expected to be additionally reduced by a factor $\sim 3$ thanks to the requirement of 2 fired gaps at the same time. In order to also take into account the projectivity condition in the inefficiency analysis, a first detailed study has been done on a simulated sample. Specific attention is payed to the identification of low energy muons, tipically large angle particles that could not fire projective pads. As shown in the table in Fig. 3, the first results are promising: for all stations the inefficiency induced by the new readout scheme is in general greater than $1 \%$ mostly for muons with energy less than $3 \mathrm{GeV}$, that would not be identified anyway by the muon PID [6]. The study is on-going in order to also take into account specific aspects such as possible contributions due to time inefficiency. 


\section{References}

72 [1] Jr. Alves et al. "The LHCb detector at the LHC". In: Journal of instrumentation 3.08 (2008), S08005 (cit. on p. 2).

74 [2] Jr. Alves et al. "Performance of the LHCb muon system". In: Journal of Instrumentation 8.02 (2013), P02022 (cit. on p. 2).

76 [3] R. Aaij et al. "Expression of interest for a phase-II LHCb Upgrade: opportunities in flavour physics, and beyond, in the HL-LHC era". In: CERN-LHCC-2017-003 (2017) (cit. on p. 2).

78 [4] F.P. Albicocco et al. "Considerations on Muon detector upgrade II”. In: LHCb-INT 007 (2020) (cit. on p. 2).

[5] G. Bencivenni et al. "The $\mu$-RWELL layouts for high particle rate". In: Journal of Instrumentation 14.05 (2019), P05014 (cit. on p. 3).

82 [6] R. Aaij et al. "Optimization of the muon reconstruction algorithms for LHCb Run 2". In: LHCb publications 007 (2017) (cit. on p. 4). 